\title{
Applications of molecularly imprinted polymers to the analysis and removal of personal care products - a review
}

\section{Figueiredo, G.L. Erny*, L. Santos and A. Alves}

\author{
LEPABE - Laboratory for Process Engineering, Environment, Biotechnology and \\ Energy, Faculdade de Engenharia da Universidade do Porto, Rua Dr. Roberto Frias, 4200- \\ 465 Porto, Portugal.
}

*Corresponding author: Tel +351-22-508-4858, E-mail: guillaume@,fe.up.pt

\author{
Abbreviations $^{1}$
}

\begin{abstract}
1 AA, acrylamide; APTES, 3-aminopropyltriethoxysilane; BA, benzoic acid; $\beta$-CD, b-cyclodextrins; BDDE, boron-doped diamond electrode; BP, benzophenone; BuP, butylparaben; BzP, benzylparaben; CE$\mathrm{AD}$, capillary electrophoresis with amperometric detection; CNDs-CS-GCE, carbon nanodots-chitosanglassy carbon electrode; CPE, carbon paste electrode; 4,4'-DCB, 4,4'-dichlorobenzophenone; DCM, dichloromethane; DLLME, dispersive liquid-liquid microextraction; 2,4-DNT, 2,4-dinitrotoluene; DVB, divinylbenzene; EDCs, endocrine disrupting compounds; EGDMA, ethylene glycol dimethylacrylate; EP, emerging pollutants; EtP, ethylparaben; EtOH, ethanol; GC, gas chromatography; GCE, glassy carbon electrode; GC-MS, gas chromatography-mass spectrometry; 2-HB; 2-hydroxybenzoic acid; 4-HBA, 4-hydroxybenzoic acid; HEMA, (Hydroxyethyl)methacrylate; HF-LPME, hollow-fibber liquid-phase microextraction; HPLC-DAD, high performance liquid chromatography with diode array detection; HPLC$\mathrm{UV}$, high performance liquid chromatography with ultraviolet detection; iBuP, isobutylparaben; iPP, isopropylparaben; LC, liquid chromatography; LC-MS/MS, liquid chromatography-tandem mass spectrometry; LLE, liquid-liquid extraction; LOD, limit of detection; MA, musk ambrette; MAA, methacrylic acid; $\mathrm{MeOH}$, methanol; MePHB, methyl p-hydroxybenzoate; MIPs, molecularly imprinted polymers; MMIP, magnetic molecularly imprinted polymers; MIP-CPE, molecularly imprinted polymercarbon paste electrode; MIP-GCE, molecularly imprinted polymer-glassy carbon electrode; MIP-ISE, molecularly imprinted polymer-ion selective electrode; MISPE, molecularly imprinted solid-phase extraction; MM, musk moskene; MMA, methyl methacrylate; MK, musk ketone; MtP, methylparaben; MT, musk tibetene; MWCNT-GCE, multiwall carbon nanotube-glassy carbon eletrode; MX, musk xylene; NIPs, non-imprinted polymers; o-phenylenediamine, o-PD; PBS, phosphate buffered saline; PCPs, personal care products; $p$-HB, $p$-hydroxybenzoic acid; $p$-HBA, $p$-hydroxyphenylacetic acid; $p$-HPPA, $p$ hydroxyphenylpropionic acid; PrP, propylparaben; PTMS, phenyltrimethoxysilane; SBSE, stir-bar sorptive extraction; SPE, solid-phase extraction; SPME, solid-phase microextraction; SA, salicylic acid; TCC, triclocarban; TCS, triclosan; TEOS, tetraethoxysilane; TPGDA, tripropylene glycol diacrylate; TRIM, trimethylolpropane; UAEM-SFO, ultrasound assisted emulsification microextraction with solidification of floating organic droplet; 2-VP, 2-vinylpyridine; 4-VP, 4-vinylpyridine; WWTP, wastewater treatment plant.
\end{abstract}




\section{Abstract}

Personal-care products (PCPs) involve a variety of chemicals whose persistency along with their constant release into the environment raised concern to their potential impact on wildlife and humans health. Regarded as emergent contaminants, PCPs demonstrated estrogenic activity leading to the need of new methodologies to detect and remove those compounds from the environment.

Molecular imprinting starts with a complex between a template molecule and a functional monomer, which is then polymerized in the presence of a cross-linker. After template removal, the polymer will contain specific cavities. Based on a good selectivity towards the template, molecularly imprinted polymers (MIPs) have been investigated as efficient materials for the analysis and extraction of the so called emergent pollutants contaminants.

Rather than lowering the limit of detections, the key theoretical advantage of MIP over existing methodologies is the potential to target specific chemicals. This unique feature, sometime named specificity (as synonym to very high selectivity) allows to use cheap, simple and/or rapid quantitative techniques such as fast separation with ultra-violet (UV) detection, sensors or even spectrometric techniques. When a high degree of selectivity is achieved, samples extracted with MIPs can be directly analysed without the need of a separation step. However, while some papers clearly demonstrated the specificity of their MIP toward the targeted PCP, such prove is often lacking, especially with real matrices, making it difficult to assess the success of the different approaches.

This review paper focusses on the latest development of MIPs for the analysis of personal care products in the environment, with particular emphasis on design, preparation and practical applications of MIPs. 


\section{Keywords}

Personal care products; emergent contaminants; estrogenic activity; molecularly imprinted polymers; wastewater. 


\section{Introduction}

Personal care products (PCPs) is a term used to refer to multiple goods that can be found in the health and beauty sections of drug and department stores and that can be bought without prescription. They include cosmetics (skin moisturizers, perfumes, lipsticks, fingernail polishes, eye and facial makeup preparations, shampoos, permanent waves, hair colours, toothpastes, and deodorants) and over the counter drugs (skin protectants such as lip balms and diaper ointments, mouthwashes marketed with therapeutic claims, antiperspirants, and treatments for dandruff or acne), or both. An example is given by antidandruff shampoo that can be used both to cleanse the hair and to treat dandruff, being regarded as a combination of cosmetic and drug [1].

As integral part of our daily life, many of the chemicals that are present in PCPs may enter the organism through direct ingestion or by absorption through the skin, where they can bio accumulated and/or be excreted by urine or simply removed by washing them off [3]. Some of the chemicals that can be found in PCPs are regarded as emerging pollutants (EP). Those are defined as any synthetic or naturally occurring chemicals or microorganisms that are not commonly monitored in the environment, with potential to enter the environment and cause known or suspected adverse ecological and/or human health effects [2]. The presence of those potential contaminants in the environment at continuously increasing concentration may be harmful to wildlife and human population. It has been clearly demonstrated for example that paraben, the most common preservative used in PCP, has estrogenic activity [4, 5]. In an epidemiologic study Kunisue, et al. [6] investigated the occurrence of five benzophenones (BP) derivatives, used in some PCP for the protection of skin and hair from UV radiation, in U.S women. The authors found that the exposure to high levels of BP UV filters could increase the odds of an endometriosis diagnosis due to its estrogenic activity. 
Because of the high levels of consumption of PCPs, those are now present in the environment at increasing concentration [7]. In Table 1 some chemicals found in PCP are presented. Those are main ingredients with environmental concern and/or the most often detected in aqueous matrices. Most of these chemicals are lipophilic (log $\mathrm{K}_{\mathrm{ow}}$ higher than 3.5), presenting a high potential of bioaccumulation [8]. They have been detected in surface waters, ground waters and drinking waters sources [9-11], as well as in several aquatic organisms in concentrations in the range of ng. $\mathrm{L}^{-1}[12,13]$. Their occurrence at such a low concentration is one of the main obstacles for their routine detection and analysis in complex samples [14]. For this reason the development of selective and economically reliable methods has become a priority.

This review focuses on the 4 families of the most used EPs in PCPs - organic UV-filters (often found in sunscreen cosmetics), preservatives (prevent decomposition in hygiene products, foods, beverages), antimicrobials (used to reduce infections, sepsis or putrefaction) and musks (used in fragrance industry in products such as laundry detergents, soaps, perfumes, etc.). Several analytical methods are available and discussed in the next section. Although those methods usually achieve analytical performances that allow quantifying such compounds the concentration step is non-selective resulting in complicated samples that can only be analysed using complex and expensive separation methods (mainly separation techniques hyphenated with mass spectrometry). Those techniques are not always compatible with routine or on-site analysis. Recently, researchers have used molecularly imprinted polymers (MIPs) and studied how they can be applied in the analysis of PCP in various matrices.

Molecular imprinting is described as a technique with the ability to produce cross-linked polymers with specific cavities that are complementary to a template molecule in terms of size, shape and functionality [15]. The imprinting process usually starts with the 
interaction between a functional monomer and the selected template in a selected solvent that acts as the porogen. The complex is then polymerized through thermal or photoinitiation in the presence of a cross-linker, producing a tri-dimensional structure. In the end the template is removed from the polymer structure leading to the creation of specific binding sites (Fig. 1).

Beyond the excellent properties exhibited by MIPs, such as high thermal and chemical stability the key advantages of these materials over other technologies are their high affinity and selectivity allowing, theoretically, to specifically remove the targeted compounds from its matrix. This is very attractive as it allows, for example, the direct analysis of the extracts without separation. This was possible for the direct determination of pentamidine in urine samples by UV spectrometry [16] or for ciprofloxacin in urine by MS spectrometry [17].

Since their appearance MIPs have been considered in several areas such as chromatographic separations [18] solid-phase extraction [19] or membrane separations [20]. However it is with chemical sensors [21] that MIP demonstrated their full potential. Those allow monitoring targeted compounds in complex environmental matrices in a fast, cheap and accurate way.

This review does not intend to give a full description of the developments of molecular imprinting which have been discussed in several recent review papers [22-24] but focusses on the preparation of imprinted materials for the recognition and extraction of EP found in personal care products. Considering a time period between 2001 and 2014 the first part of this review addresses to the development of MIPs that are selective to compounds included in the four classes of chemicals widely used in PCPs - organic UVfilters, preservatives, antimicrobials and musks (Table 1). The second part of the review analyses the applications and advantages of using this MIPs compared to the traditional 
methods available for the analysis of PCPs.

To the best of the authors' knowledge, this is the first review on imprinting technology applied to personal care products.

\section{MIP synthesis for personal care products}

PCP include, among other, organic ultraviolet filters, preservatives, antimicrobials and musk fragrances [25]. These chemicals have been found in concentrations ranging between tens to thousands of ng in environmental solid [26] and liquid samples [3]. Recently, Haman, et al. [27] concluded that in the parabens family, methylparaben and propylparaben were the most often detected in wastewater influents with mean concentrations up to 40 and $14 \mathrm{nM}$, respectively. As for the other classes of PCPs, Luo, et al. [28] when studying the occurrence of micropollutants in aquatic environment, concluded that galaxolide and tonalide were the most frequent musks in influents showing concentrations of 0.12 to $97 \mathrm{nM}$. Triclosan for antimicrobials and benzophenone- 3 for organic UV filters were found in concentrations ranging from 0.10 to 83 and 0.43 to 5 $\mathrm{nM}$, respectively [28].

The complexity exhibited by the matrices containing these contaminants is one of the great challenges for their quantification. For an accurate and precise analysis, samples need to be previously treated in order to concentrate the analyte and/or remove potential interferents and increase the sensitivity and selectivity of the analytical method. The search for new alternatives led researchers to study MIPs for the analysis of chemicals contained in PCPs. Those are regarded as versatile sorbents whose selectivity can be tuned towards a target molecule. Moreover these polymers are characterized by high stability and ease of production [29]. However, despite the advantages of imprinted polymers, there's still a reduced number of studies involving MIPs and PCPs. To the best 
of author's knowledge for a period between 2001 and 2014, 19 papers were published involving the synthesis of imprinted polymers for the analysis or the extraction of PCPs (13 for preservatives, 4 for antimicrobials, and 1 for both UV filters and musks). Table 2 summarises the key findings from those works, including the target analytes and matrices, the choice of templates, monomers and cross linkers, the analytical methodologies used and the analytical performances achieved. Those works were further considered in statistical analysis concerning the most important variables for the synthesis of PCPs based MIPs.

\section{Template}

The success of the molecular imprinting process is determined by the quality of the interaction between the functional monomer and the template. Ideally those interactions should be strong so that the recognition mechanism after the synthesis of the polymer could be enhanced. Suitable species should be chosen carefully in order to produce the highest number of well-defined binding sites [30].

Based on the non-covalent approach used for the synthesis of the MIPs presented on Table 2, the main considerations involved in the choice of template are its stability, the ability to establish hydrogen bonding and the absence of polymerizable groups to avoid reaction with the newly free radicals. The imprinting molecule should also share a similar structure with the analyte so that recognition could be improved [31]. While the target analytes may seem a good choice for the template, many publications highlighted the difficulty to achieve a complete removal of the template even after extensive washing procedures [32]. As a consequence not only a decrease of the available binding sites is observed but also a slow release of the remaining template, in particular during trace analysis, affecting negatively the final results [33]. The use of a template with similar 
structure to the target analyte (dummy strategy) allows overcoming problems due to the template leakage. However, it must be verified that the signal produced by the dummy molecule will not interfere in the quantification [33].

Although authors are aware of the disadvantages when using the analyte as template, $84 \%$ of the PCPs based MIPs were produced using the same molecule as target and template. For example, Liang, et al. [34], prepared a MIP for the analysis of triclosan in toothpaste samples using triclosan as template. Regarding the risks of template leakage during applications, no information was found in the analysed reports with concern to the extent of the extraction. As demonstrated by Zhongbo et al. [35] the mass balance can be used to verify that most of the template has been removed. This is done by first quantifying the amount of template that has been trapped inside the MIP during formation. The amount of template removed during washing is then measured. Those simple steps allow to quantify the amount of template that remained trapped.

In contrast, the dummy approach was not so explored in the considered reports involving MIPs and PCPs. An example is described by Lopez-Nogueroles, et al. [36], that selected 2,4-dinitrotoluene (2,4-DNT) for the preparation of an imprinted sorbent for the analysis of nitro musks, because 2,4-DNT shows a structural analogy with the target nitro-musks.

\section{Functional Monomer}

The selection of the monomer plays an important role on the final properties of the polymer. It should be able to interact with the template through covalent or non-covalent interactions. The latter, the most common procedure for the synthesis of MIPs, demands a careful choice due to the lower intensity of the interactions involved [37]. The literature on MIPs provides an extended list of the monomers suitable for each approach [38], but methacrylic acid (MAA), acrylic acid (AA) and 2- or 4-vinylpyridine (2- or 4-VP) are the 
most common choices for molecular imprinting. Among these, MAA is often selected due to the possibility to interact with the template through both non-covalent and covalent interactions [39].

It was observed that in the 19 studies analysed in this review, 35\% of the imprinted polymers were prepared with MAA, particularly for analysis of parabens [40]. However other monomers were successfully employed in the synthesis of MIPs selective to parabens, namely 4-VP [41] and acrylamide [42] (Fig. 2). Núñez, et al. [43] used MAA and 4-VP to prepare different polymers to be used in a molecularly imprinted solid-phase extraction (MISPE) procedure. Polymers were evaluated according to their recognition properties towards benzylparaben, revealing that while both MAA and 4-VP could strongly interact with the template, the inclusion of MAA provided a higher number of selective binding sites which results in a clear imprinting effect.

With the development of the imprinting techniques other monomers have been tested, among which 3-aminopropyltriethoxysilane (APTES), phenyltrimethoxysilane (PTMS) and o-phenylenediamine (o-PD). Employed in 31\% of the considered studies the selection of these chemicals is highly dependent on the preparation method.

Gao, et al. [44] produced imprinted polymers for the extraction of triclosan (TCS) in environmental waters. The authors evaluated the adsorption capacity and the imprinting effect of the polymers by using APTES, PTMS and a combination of these as functional monomers. The results showed that APTS lead to a better polymer compared with the ones made with PTMS and APTES+PTMS. This was attributed to the presence of an amine group in APTES which had the ability to establish hydrogen bonding with the oxygen atom and interact with hydroxyl group in TCS. On the other hand PTMS provides the phenyl group for $\pi-\pi$ interaction with the phenyl residue in TCS which are less intense than the interactions between APTS and TCS. As a result, APTES was chosen as 
functional monomer in their subsequent work.

\section{Cross-linker}

The cross-linking agent is responsible for the morphology of the polymeric material, the stabilization of the binding cavities and to ensure the mechanical stability of the polymer. Many reviews have highlighted its importance providing lists of cross-linkers that are compatible with molecular imprinting [31, 38]. Ethylene glycol dimethacrylate (EGDMA), trimethylolpropane trimethacrylate (TRIM) and divinylbenzene (DVB) are the most common cross-linkers for MIPs production. When it comes to the application of MIPs for the analysis of PCPs, EGDMA has been largely used as cross-linker (Fig 3). TEOS has also been significantly employed, despite being exclusively suitable for the sol-gel process. Other cross-linkers like DVB and TRIM have been used in mixture to produce imprinted nanobeads for the analysis of triclosan [34]. The authors demonstrated that the use of these two cross-linkers allowed a precise control of imprinted particles size. Triclosan imprinted nanobeads were obtained with a high degree of homogeneity exhibiting diameters in a range of 200 to $300 \mathrm{~nm}$ which were suitable for sensor purposes [34].

\section{Polymerization procedures}

Bulk polymerization is the most common procedure to obtain imprinted microspheres. In this procedure, all species, monomer, cross-linker, template and radical initiator are initially dissolved in the porogen. Prior to the polymerization either by thermal of photochemical initiation, the reaction media is purged with nitrogen for oxygen removal whose presence can retard free radical polymerization. In the end, a monolith is obtained and submitted to mechanical processes of grinding and sieving, resulting in irregular 
particles with diameter between 10-25 $\mu \mathrm{m}$. However, despite the simplicity and reliability of this technique, particles with irregular shape and the high amount of waste produced (up to $80 \%$ ) are significant drawbacks associated to the bulk technique [37]. As alternative to bulk polymerization, new methods have been proposed to better control particles size and porosity. Suspension polymerization is seen as a simple method capable of producing homogeneous particles exhibiting sizes in the tens of $\mu \mathrm{m}$. In this approach, the reaction mixture is dispersed onto an immiscible phase, typically water, and polymerization is carried out inside the droplets. [15]. Mayes and Mosbach [45] also tested a perfluorocarbon liquid as dispersing agent in a suspension protocol. The latter provided an inert phase that was immiscible with most organic phases used in the suspension polymerization. Precipitation polymerization is ideal to prepare MIP microspheres with sizes between $\mathrm{nm}$ and $\mu \mathrm{m}$. The reaction medium comprises monomer, template, cross-linker and initiator which are dissolved in a considerable volume of porogenic solvent. During this process the polymeric chains start to grow, becoming insoluble in the porogen and consequently start to precipitate [46]. However, as reported by Chen, et al. [29], the large volume of porogenic solvent required for this method is a considerable disadvantage as it generates increased costs and environmental concerns.

Another method that has been used to prepare imprinted polymers is the multistep swelling polymerization. This technique produces monodispersed particles with final size that is slightly higher than the ones prepared by precipitation. Compared to other methods it is considered a time-consuming process and like suspension protocol, it uses water as continuous phase which will be responsible for the lower performance of the final product [29] .

An interesting comparative study was performed by Pérez-Moral and Mayes [48], who imprinted propranolol by different polymerization methods, namely, bulk, suspension, 
emulsion, two-step swelling and precipitation. After performing the template extraction the resulting polymers were submitted to adsorption assays in the presence of propranolol. The amount of rebinding analyte was determined in different media, organic and aqueous. Results showed that precipitation based particles had a better performance in organic media, while two-step swelling registered the highest binding ratio in water.

In the 1980s the sol-gel method arises as new technique to prepare molecular imprinted particles in silica. Sol-gel MIPs are prepared by the hydrolysis and polycondensation of silane based monomers such as tetramethoxysilane (TMOS) or tetraethoxysilane (TEOS) which form highly cross-linked silica materials. As advantages this method shows a low reactivity in extreme conditions but most important the highly cross-linked structure of silica leads to more stable cavities compared to more flexible organic polymers. However despite being highly porous, the diffusion pathaways are not direct in silica MIPs. Also as a result of a smaller distance between cross-links, the swelling in imprinted silica materials is much lower compared to the organic MIPs [47].

Regarding the studies analysed in this review it was observed that PCPs based imprinted polymers have been mostly prepared using precipitation polymerization (Fig 4). This procedure allows an excellent control over the particle size leading to uniform and spherical particles. Bulk polymerization was also reported [49], as well as surface imprinting [41] and sol-gel [36]. These latter have been considered for the easiness by which the template is removed and for enabling the synthesis of water-compatible MIPs, respectively. In addition to the previous polymerization methods electropolymerization has also been applied to prepare MIP based chemical sensors to the analysis of PCPs [50]

Water compatibility 
The water compatibility of MIPs is one of the main concerns. The polymer shell of the structure if often hydrophobic and MIP if used in aqueous media will often have multiple sorption mechanisms. While the core of the MIP will provide molecular recognition, various molecules, including the target molecules may interact with the outer layer via hydrophobic interactions in a similar way as with classical polymeric sorbent. This may ruin the high selectivity of the MIP but also induce problem of contamination of the surface.

While not yet used with PCPs, new strategies have been tested to improve the water compatibility. For instance, this can be done by using hydrophilic monomers like $\beta$ cyclodextrins $\beta-\mathrm{CD}[51,52]$ and (Hydroxyethyl)methacrylate HEMA $[53,54]$.

\section{Use of molecularly imprinted polymers in analysis of PCPs}

\section{MIP as sorbent for Solid-Phase Extraction (SPE)}

Over the years a large number of reports have described the application of MIPs as selective sorbents for SPE technique (MISPE). In the case of PCPs near 50\% (of the 19 publications analysed in this study) of the MIPs were applied as sorbents for a SPE technique. Environmental waters [55], soils [49], sediments [43], plant extracts [42] and soy [56], comprise the different samples that were successfully treated by MISPE. Beltran, et al. [57] prepared a MIP in the monolithic form using butylparaben as template. The imprinted polymer was used as sorbent in a SPE procedure for the analysis of parabens in environmental water. Results were compared with the performance of a commercial sorbent (HLB Oasis). The extraction with MISPE allowed a higher recovery and a cleaner chromatogram, however only butylparaben and benzylparaben were detected with recoveries of 58 and $27 \%$, respectively (Fig. 5). Concerning the other 
parabens in analysis, methylparaben and ethylparaben were not quantified due to peak interference. In addition, the MISPE protocol was optimized for $500 \mathrm{~mL}$ of sample, which is unlikely to see in these procedures. The method exhibited low recoveries which could be due to the procedure adopted in the MIP preparation. The mechanical processes involved in bulk polymerization are responsible for destroying the polymeric cavities resulting in a reduced affinity towards the analyte.

As an alternative Lorenzo, et al. [55] applied the precipitation protocol to prepare a selective MIP towards butylparaben. This work combined a MISPE protocol with the electrochemical detection by glassy carbon electrodes modified with multi-wall carbon nanotubes dispersed in nafion. This methodology led to good recoveries ranged between 82 and $85 \%$ for $100 \mathrm{~mL}$ of sample containing different concentrations of butylparaben providing a limit of detection of $3.8 \mu \mathrm{g} . \mathrm{L}^{-1}$. These limits are however higher than the one obtained with methodologies like SPE coupled with UHPLC-MS ${ }^{2}$ [58] or SPME coupled with GC-MS ${ }^{2}$ [59] that provided limits of 0.1 and 5.7 ng.L - $^{-1}$, respectively. Despite the improvement compared to the method reported by Beltran, et al. [57], the authors observed a considerable interference on the final recoveries of butylparaben, when other parabens were added into water samples. In fact the measured recoveries increased to $150 \%$, fact that was assigned to an overlapping signal. The authors concluded that the parabens could not be distinguished, and as consequence the measured signal should be interpreted as the total amount of parabens in the sample. This example clearly highlights the potential problem with MIP design. In this example, methylparaben, ethylparabem and benzylparaben only differ by their alkyl group. While theoretically, MIP should be more selective to the template (here benzylparaben), due to size recognition, this effect is very small compared to hydrogen bonds. Designing a MIP that can recognised on particular paraben is a very complicate task. An interesting comparison between the 
performance of MIP and commercial C18 SPE sorbent was presented by Verma and Xia [49] for the analysis of triclosan and triclocarban (TCC) in soil and biosolids. The bulk polymer was imprinted with 4,4'-dichlorobenzophenone (4,4'-DCB) a dummy template, after being confirmed that there was no interference in the quantification of TCS and TCC. SPE procedures for each sorbent were previously optimized and the analysis of the extracts was carried out by HPLC-UV. In the particular case of biosolids it was proved that MISPE lead to a better extraction of TCC- $89.9 \%$ in comparison with the $43.3 \%$ obtained with the $\mathrm{C} 18$ sorbent. As for TCS interference in the chromatogram after extraction with the $\mathrm{C} 18$ cartridge made the analysis unreliable. On the other hand, MISPE confirmed its selectivity leading to a much clearer chromatogram which resulted in a recovery of $82.0 \%$ for TCS. In comparison to other methods developed for the analysis of TCS in biosolids, the presented combination of MISPE with HPLC provides higher limits of detection. Nevertheless for quantification methods involving LC-MS [60] or GC-MS [61] the limits of detection can be at least 60 times lower than those obtained by MISPE-HPLC-UV, this principally because of the detection mode. While, the high selectivity provide by MIP is no key with those instruments, MISPE can also be very advantageous in conjunction with LC-MS, as the extensive sample clean-up allow to reduce ion suppression usually associated with complex samples [41] [25].

\section{Nanoparticle MIPs}

Conventional imprinted polymers present, however, some limitations like slow mass transfer or incomplete template removal which negatively affects the number of available sites for rebinding. New initiatives to overcome those limitations have been reported such as the combination of imprinting technique with nanomaterials for faster rebinding kinetics and higher binding capacity. 
Gao, et al. [44] reported the synthesis of a core-shell imprinted polymer for the extraction of triclosan from environmental water samples. In this report the initial imprinted shells were anchored onto the surface of carbon nanotubes via a surface molecular imprinting sol-gel process. The obtained nanoparticles were added to $50 \mathrm{~mL}$ of spiked water sample for the removal of triclosan. After the adsorption process, polymer was separated by centrifugation and eluted with a proper solvent. The extracts were then quantified by HPLC which provided recoveries from 90.7 to $95.3 \%$.

There has also been an interest in the encapsulation of particles with magnetic properties. In this format, the magnetic molecularly imprinted polymers (MMIP) can easily be isolated from the sample by applying a magnetic field. Magnetic particles are based on $\mathrm{Fe}_{3} \mathrm{O}_{4}$ characterized by a low toxicity, low cost and eco-friendliness. Shi, et al. [41] have synthesized two magnetic imprinted polymers for the extraction of PCPs (hydrobenzoic acids) from aqueous solutions. Firstly, the authors prepared the $\mathrm{Fe}_{3} \mathrm{O}_{4}$ microspheres which were then covered by silica. After that, the templates, benzoic acid and 4-hydroxybenzoic acid (4-HBA), were left in interaction with the monomer before polymerization at the surface of the magnetic microspheres. The analysis of the water samples was carried out by the suspension of the imprinted particles in $40 \mathrm{~mL}$ of sample. Mixture was then agitated during the adsorption process and in the end particles were removed by a magnet. Extracts were then analysed by HPLC leading to recoveries between 83.2 and $103 \%$ and limits of detection from 7 to $160 \mathrm{nM}$.

\section{MIP based sensors}

The application of MIPs in the field of chemical sensors has received a special attention in the recent years. MIP based sensors have been discussed as promising materials for different fields such as clinical, bioanalytical, process control and environmental 
applications [62]. A sensor itself is defined as a device that comprises a recognition element and a transducer that converts the chemical information into measurable signal [63]. Experts believe that in a near future MIP based sensors will compete with analytical techniques like liquid chromatography, mass spectrometry, spectroscopic methods among others [64]. MIP sensors offer advantages like low cost of manufacture, easy storage, extended lifetime and the capacity of being applied in critical conditions. For such applications, the imprinted polymer is coupled with a transducer which converts the sensing properties of the MIP into a measurable signal. The sensivity of the resulting sensor is directly affected by the affinity shown by the imprinted polymer towards the analyte. Therefore it is desirable to use imprinted particles in sizes of micro or nanometeres with high surface-to-volume ratio. Another critical aspect involved in the preparation of a MIP based sensor is on how to integrate the polymer with the transducer. With that purpose several methodologies have been developed: (i) in-situ method like Electropolymerization, (ii) drop-casting of pre-formed polymers, (iii) design of composite membranes containing conducting materials, MIP and a binder, (iv) in-situ polymerization of the monomer and (v) self-assembly of monolayers.

Regarded as the most commonly used method to prepare electrochemical sensors, electropolymerization allows the synthesis of an imprinted film directly on the surface of the electrode. The quality of the film is determined by several factors (e.g. type of electrode, current density, temperature, presence of water, etc.). However it has the advantage of placing the polymer at a precise spot of the detector surface.

Liu, et al. [65], made the first attempt in the development of a MIP based sensor for the analysis of triclosan. The sensor was prepared by the electropolymerization of ophenylenediamine (o-PD) on a glassy carbon electrode (GCE) in the presence of template. The method provided satisfactory results even in the presence of similar structures to the 
analyte with a limit of detection of $8.0 \times 10^{-8}$ mol.L-1. More recently, Li, et al. [50], produced an amperometric benzophenone sensor based on MIP through the electropolymerization of $o$-phenylenediamine (o-PD) on a GCE. The sensor was successfully applied to the analysis of benzophenone, an organic UV-filter, in food packaging material samples previously extracted with ethanol. The levels of benzophenone found in plastics for cake, bakery products and milk were ranged between 0.2 and $0.40 \mathrm{mg} . \mathrm{dm}^{-2}$ which were in agreement to the ones obtained for HPLC-UV indicating that these kinds of sensors present an attractive alternative to more expensive analytical methods.

Another method for immobilizing the polymer onto the surface of the electrode was developed by Wang, et al. [66]. The authors prepared a polymeric solution with two templates (methylparaben and propylparaben) and then dropped it onto the surface of the GCE. After covering the electrode with a piece of slip, the mixture was polymerized on an oven forming a transparent polymer film. By using a strategy of a dual template the authors were able to determine the total content of four kinds of parabens in cosmetic samples $(14.5$ to $20.3 \mu \mathrm{M})$. The combination of MIP based sensor with a strategy based on the impression of multi-templates seems to be promising in the detection and separation of homologous compounds in the same sample.

Also for the analysis of parabens, Gholivand, et al. [67] prepared imprinted nanoparticles via precipitation polymerization which were subsequently incorporated into the carbon paste electrode (CPE) by mixing different percentages of graphite, solid binding matrix (n-eicosane) and imprinted polymer. It was observed that in the presence of other interferents, the signal of propylparaben remained unchanged. The results showed good recoveries of the analyte in cosmetic samples with values between 97.3 and $103 \%$. In this study the authors compared the performance of the sensor with other sensors 
for propylparaben. MIP-GCE sensor exhibited the lowest limit of detection showing the excellent sensitivity of the method.

The use of imprinted polymers has remarkably improved the sensitivity of the conventional chemical sensors. Table 3 lists the recent reports on the analysis of PCPs by sensors. A direct comparison between the potentiometric sensors revealed that MIP based sensors provided lower limits of detection. As for the recoveries, these latter MIP sensors showed values in agreement with the other methodologies. Therefore it is predictable that the number of publications on MIP sensors should increase intensely in the future as a consequence of the promising results obtained so far.

The overall analysis of the methods involving the synthesis of MIPs with selectivity towards PCPs showed that parabens have been the main target of molecular imprinting. On the other hand, molecular imprinting is still less developed for the other classes of PCPs (antimicrobials, musks and UV filters). SPE have been the preferred applications for PCPs based MIPs, but successful approaches have emerged with the inclusion of imprinted polymers in chemical sensors for the analysis of PCPs.

\section{Conclusions}

The information provided in this review highlights the concern about the impact of PCPs in humans and wildlife on a variety of environmental and biological samples.

In recent years several analytical methods have been developed for the extraction and determination of these compounds. Methods like the classical SPE and microextraction 
techniques coupled with powerful analytical techniques (GC-MS and LC-MS) have allowed researchers to reach lower limits of detection in the determination of PCPs.

More recently high selective materials (MIPs) have become an alternative to existing methods for the analysis of PCPs. The use of imprinted polymers as selective sorbents for the SPE technique has proven to be valuable for the extraction of one or more contaminants.

In addition the application of the MISPE technique to the extraction of PCPs have increased the selectivity of the method without using complex techniques based on mass detectors. Innovations like MIP based sensors have also been introduced for the analysis of PCPs, providing a promising alternative to the chromatographic methods due to the reduced time of analysis and cost of production. MIPs have also been tested in batch experiments for the removal of PCPs from environmental waters. These attempts have increased the expectation for a future application of MIPs on wastewater treatment. New methodologies for the preparation of MIPs have been studied so that the limitations like slow mass transfer, adsorption capacity, template leakage and reduced water compatibility could be overcome. However there's still a long way to go and many challenges lie ahead before MIPs could successfully be applied in water analysis and purification.

\section{Acknowledgments}

This work was funded by FEDER funds through the Operational Program for Competitiveness Factors - COMPETE, ON.2 - O Novo Norte - North Portugal Regional Operational Program and National Funds through FCT - Foundation for Science and Technology under the projects: PEst-C/EQB/UI0511, NORTE-07-0124-FEDER-000025 
- RL2_ Environment\&Health , SFRH/BD/86941/2012 and IF/00528/2013. 


\section{Figures captions}

Figure 1. Schematic representation of the molecular imprinting process. Reproduced from García-Calzón and Díaz-García [68], with permission.

Figure 2. Type of functional monomer used in 19 studies regarding the development of MIPs for the analysis PCPs. AA, acrylamide; APTES, 3-aminopropyltriethoxysilane; MAA, methacrylic acid; MMA, methyl methacrylate; o-PD, o-phenylenediamine; PTMS, phenyltrimethoxysilane; 4-VP, 4-vinylpyridine.

Figure 3. Type of cross-linker used in 19 studies regarding the development of MIPs for the analysis PCPs. DVB, divinylbenzene; EGDMA, ethylene glycol dimethylacrylate; TEOS, tetraethoxysilane; TPGDA, tripropylene glycol diacrylate; TRIM, trimethylolpropane trimethacrylate.

Figure 4. Polymerization methodologies applied in 19 studies regarding the development of MIPs for the analysis PCPs.

Figure 5. High performance liquid chromatography with ultraviolet detection (HPLCUV) chromatograms of water samples after the extraction with MIP and C18 by solidphase procedures. Before extraction samples were spiked with methylparaben (MtP), ethylparaben (EtP), butylparaben (BuP) and benzylparaben (BzP). Reproduced from [57], with permission. 


\section{List of Tables}

Table 1. Name, physical-chemical properties and use for some of the main compounds that can be found in personal care products.

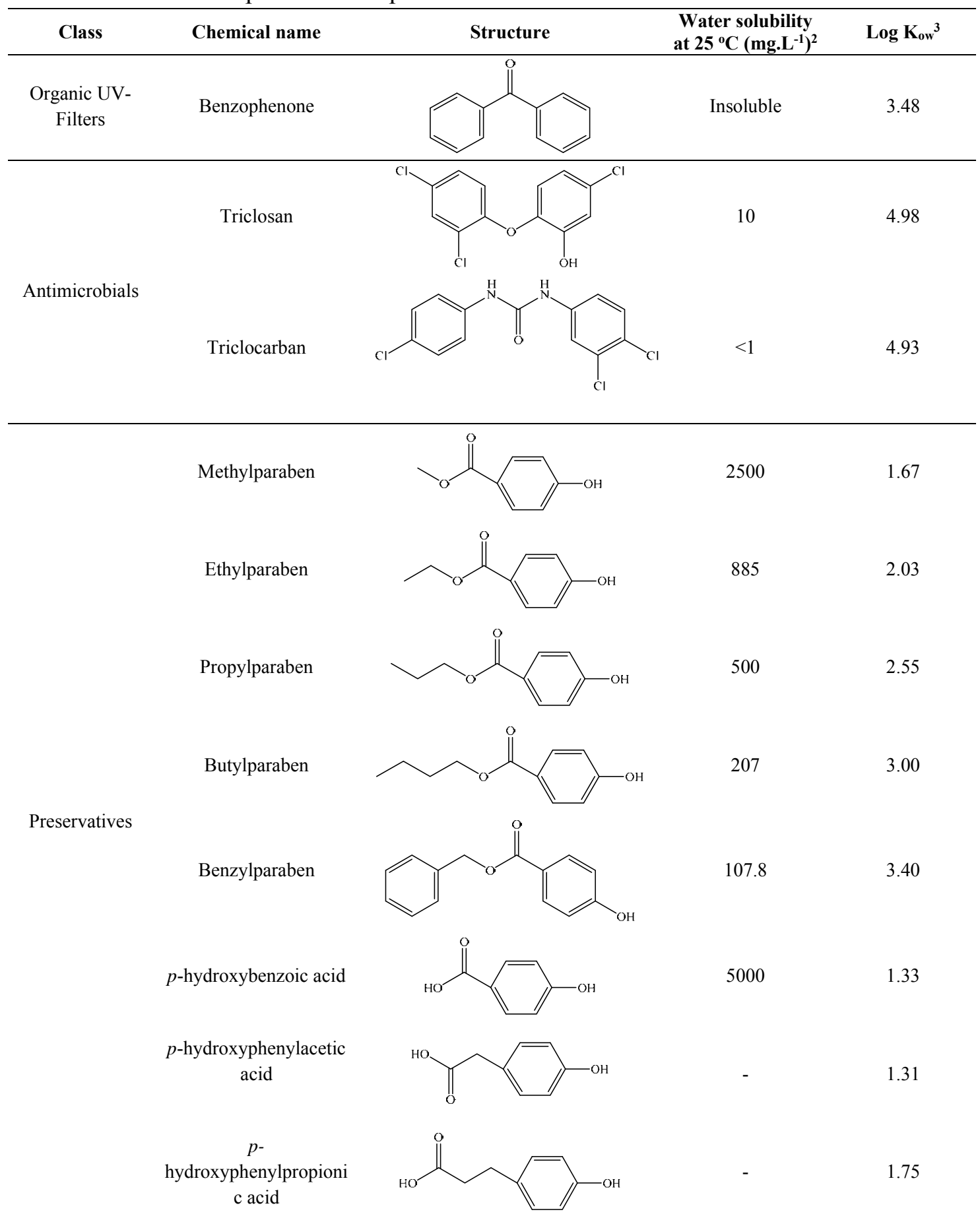

${ }^{2}$ Values from PubChem Compound (http://www.ncbi.nlm.nih.gov/pccompound)

${ }^{3}$ Values from Chemspider (www.chemspider.com) 


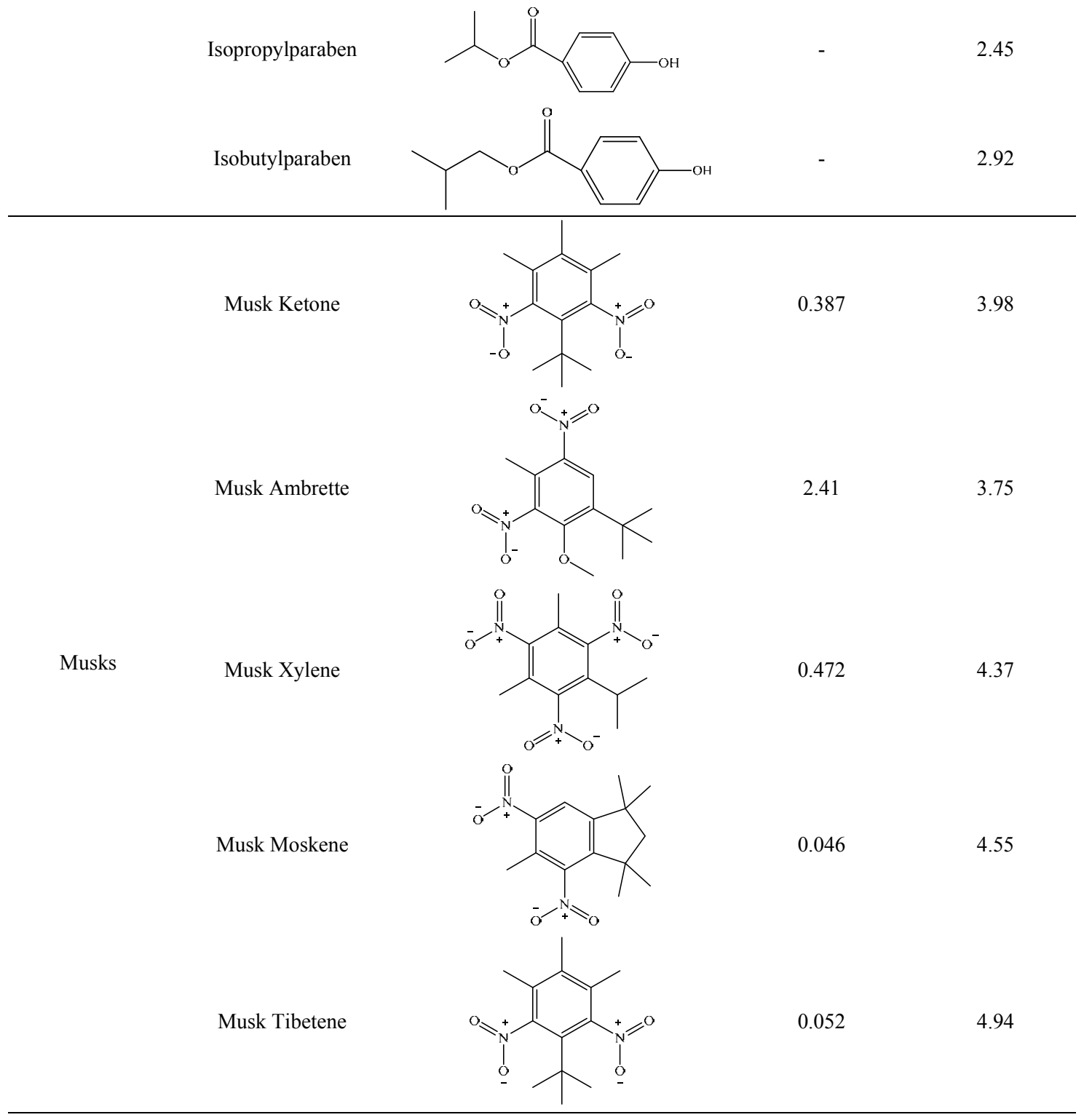




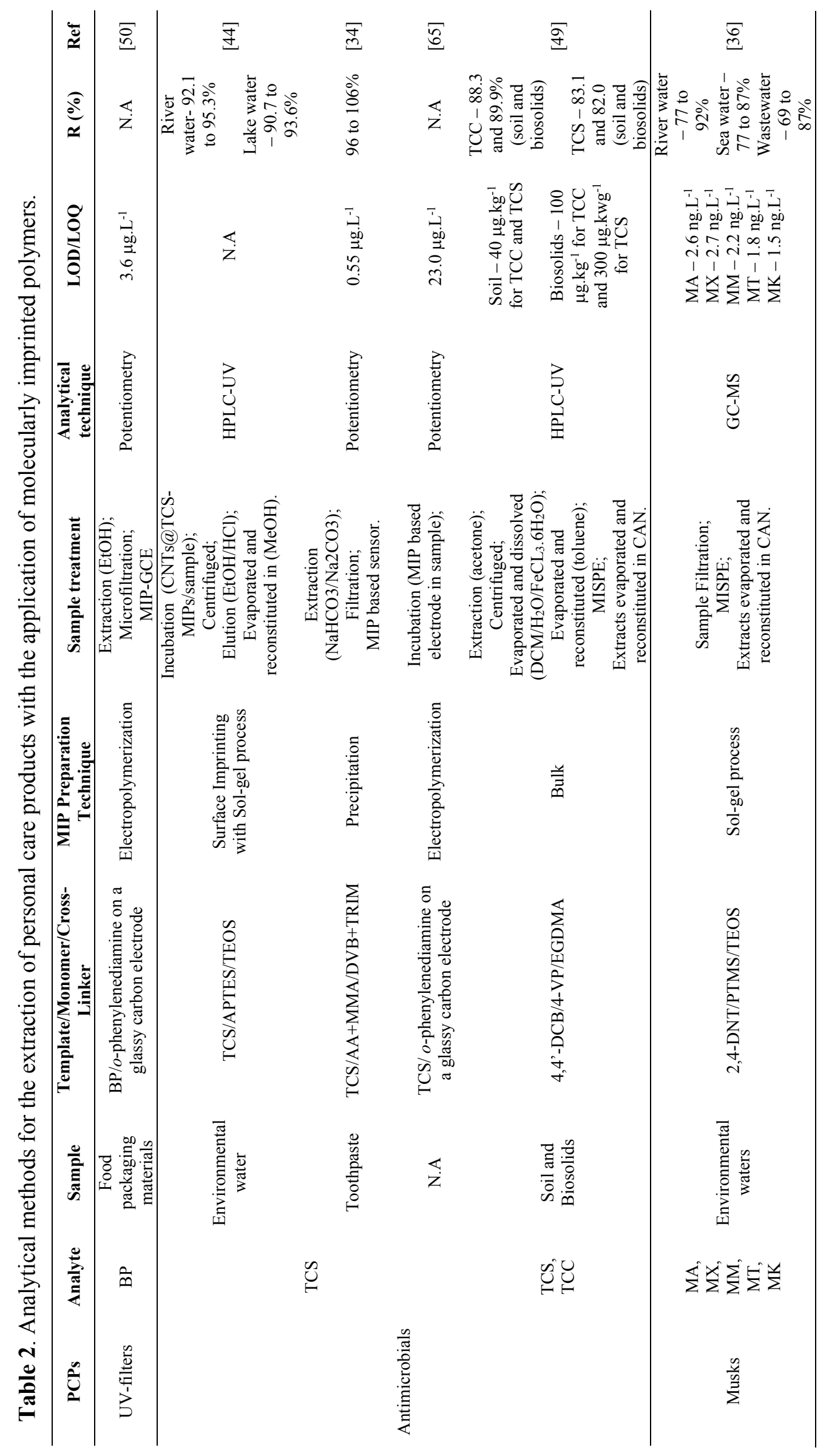




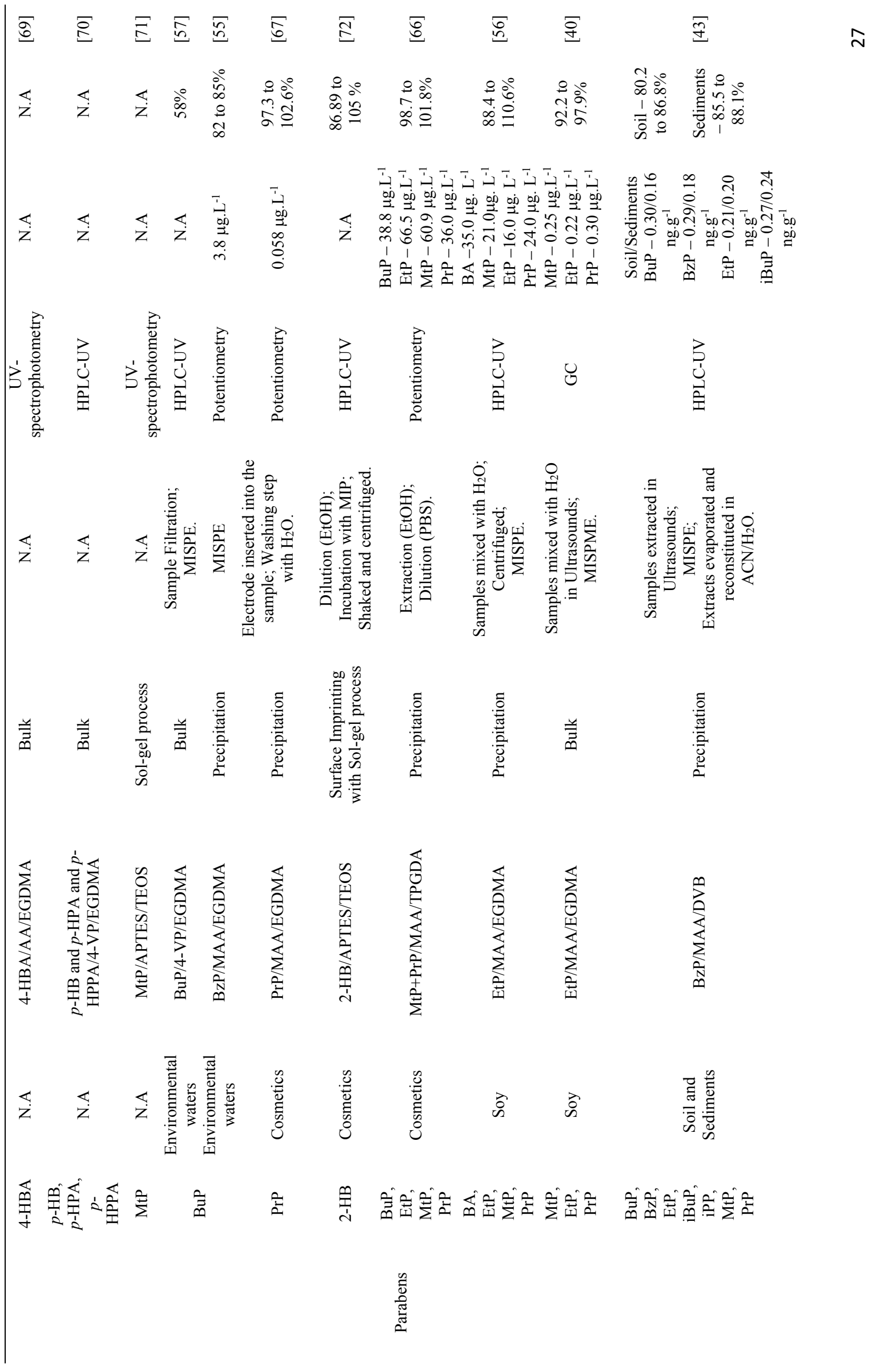




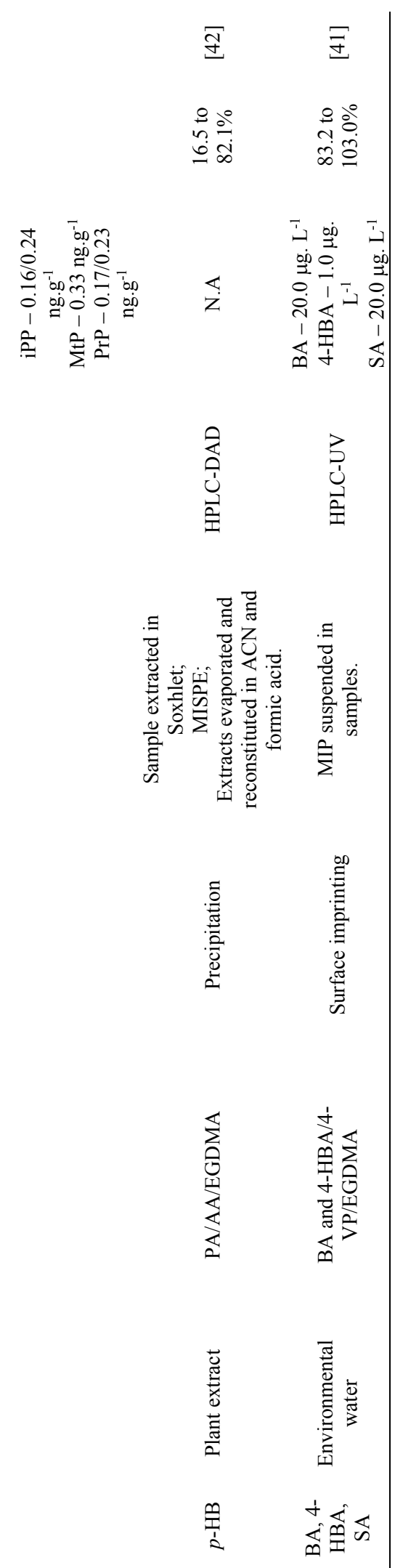


Table 2. Comparison between MIP based sensors and the current sensors developed for the analysis of PCPs.

\begin{tabular}{|c|c|c|c|c|c|c|}
\hline $\begin{array}{c}\text { Sample } \\
\text { treatment }\end{array}$ & Analyte & Matrix & Sensor & $\begin{array}{l}\text { LOD } \\
\left(\mu \mathrm{g} . \mathrm{L}^{-1}\right)\end{array}$ & $\mathbf{R}(\%)$ & Ref \\
\hline Dilution & \multirow{3}{*}{ PP } & $\begin{array}{l}\text { Aqueous } \\
\text { solutions }\end{array}$ & $\begin{array}{c}\text { BDDE } \\
\text { (potentiometric) }\end{array}$ & 174.8 & N.A & [73] \\
\hline $\begin{array}{l}\text { Dilution or } \\
\text { SPE }\end{array}$ & & Food & $\begin{array}{c}\text { CE-AD } \\
\text { (amperometric) }\end{array}$ & 27.57 & 84.0 & [74] \\
\hline $\begin{array}{c}\text { Dilution and } \\
\text { Sonication }\end{array}$ & & Cosmetics & $\begin{array}{c}\text { MIP-CPE } \\
\text { (potentiometric) }\end{array}$ & 0.058 & $\begin{array}{c}97.3- \\
102.57\end{array}$ & [67] \\
\hline $\begin{array}{l}\text { Sonication } \\
\text { and Dilution }\end{array}$ & \multirow{3}{*}{ TCS } & \multirow{3}{*}{ Toothpaste } & $\begin{array}{l}\text { MWCNT-GCE } \\
\text { (potentiometric) }\end{array}$ & 16.50 & $\begin{array}{l}97.4- \\
107.4\end{array}$ & [75] \\
\hline $\begin{array}{l}\text { Dilution and } \\
\text { Sonication }\end{array}$ & & & $\begin{array}{l}\text { CNDs-CS-GCE } \\
\text { (potentiometric) }\end{array}$ & 2.66 & 117.4 & [76] \\
\hline Dilution & & & $\begin{array}{c}\text { MIP-ISE } \\
\text { (potentiometric) }\end{array}$ & 0.55 & $96-106$ & [34] \\
\hline \multirow[t]{2}{*}{$\begin{array}{l}\text { Dilution and } \\
\text { Sonication }\end{array}$} & \multirow{2}{*}{$\mathrm{BPH}$} & Sunscreens & $\begin{array}{c}\text { BDDE } \\
\text { (potentiometric) }\end{array}$ & 24.96 & $\begin{array}{l}97.2- \\
98.1\end{array}$ & [77] \\
\hline & & $\begin{array}{c}\text { Food packaging } \\
\text { materials }\end{array}$ & $\begin{array}{c}\text { MIP-GCE } \\
\text { (potentiometric) }\end{array}$ & 3.46 & N.A & [50] \\
\hline
\end{tabular}




\section{References}

[1] FDA, Are all "personal care products" regulated as cosmetics?, Food and Drug Administration, 2015.

[2] S. Solak, N. Vakondios, I. Tzatzimaki, E. Diamadopoulos, M. Arda, N. Kabay, M. Yüksel, A comparative study of removal of endocrine disrupting compounds (EDCs) from treated wastewater using highly crosslinked polymeric adsorbents and activated carbon, J. Chem. Technol. Biotechnol., 89 (2014) 819-824.

[3] M. Pedrouzo, F. Borrull, R.M. Marcé, E. Pocurull, Analytical methods for personal-care products in environmental waters, TrAC, Trends Anal. Chem., 30 (2011) 749-760.

[4] R.J. Witorsch, J.A. Thomas, Personal care products and endocrine disruption: A critical review of the literature, Critical Reviews in Toxicology, 40 (2010) 1-30.

[5] R. Golden, J. Gandy, G. Vollmer, A review of the endocrine activity of parabens and implications for potential risks to human health, Critical Reviews In Toxicology, 35 (2005) 435458.

[6] T. Kunisue, Z. Chen, G.M. Buck Louis, R. Sundaram, M.L. Hediger, L. Sun, K. Kannan, Urinary Concentrations of Benzophenone-type UV Filters in US Women and Their Association with Endometriosis, Environmental science \& technology, 46 (2012) 4624-4632.

[7] J. Regueiro, M. Llompart, E. Psillakis, J.C. Garcia-Monteagudo, C. Garcia-Jares, Ultrasoundassisted emulsification-microextraction of phenolic preservatives in water, Talanta, 79 (2009) 1387-1397.

[8] I. Jiménez-Díaz, A. Zafra-Gómez, O. Ballesteros, A. Navalón, Analytical methods for the determination of personal care products in human samples: An overview, Talanta, 129 (2014) 448-458.

[9] V. Matamoros, E. Jover, J. Bayona, Advances in the determination of degradation intermediates of personal care products in environmental matrixes: a review, Anal Bioanal Chem, 393 (2009) 847-860.

[10] P. Cuderman, E. Heath, Determination of UV filters and antimicrobial agents in environmental water samples, Anal Bioanal Chem, 387 (2007) 1343-1350.

[11] J.-Q. Jiang, Z. Zhou, V.K. Sharma, Occurrence, transportation, monitoring and treatment of emerging micro-pollutants in waste water - A review from global views, Microchem. J., 110 (2013) 292-300.

[12] H.-R. Buser, M.E. Balmer, P. Schmid, M. Kohler, Occurrence of UV Filters 4Methylbenzylidene Camphor and Octocrylene in Fish from Various Swiss Rivers with Inputs from Wastewater Treatment Plants, Environ. Sci. Technol., 40 (2006) 1427-1431.

[13] M. Bachelot, Z. Li, D. Munaron, P. Le Gall, C. Casellas, H. Fenet, E. Gomez, Organic UV filter concentrations in marine mussels from French coastal regions, Sci. Total Environ., 420 (2012) 273-279.

[14] D.L. Huang, R.Z. Wang, Y.G. Liu, G.M. Zeng, C. Lai, P. Xu, B.A. Lu, J.J. Xu, C. Wang, C. Huang, Application of molecularly imprinted polymers in wastewater treatment: a review, Environ Sci Pollut Res Int, DOI 10.1007/s11356-014-3599-8(2014).

[15] V. Pichon, F. Chapuis-Hugon, Role of molecularly imprinted polymers for selective determination of environmental pollutants-A review, Anal. Chim. Acta, 622 (2008) 48-61.

[16] B. Sellergren, Direct Drug Determination by Selective Sample Enrichment on an Imprinted Polymer, Anal. Chem., 66 (1994) 1578-1582.

[17] E. Caro, R.M. Marcé, P.A.G. Cormack, D.C. Sherrington, F. Borrull, E. Caro, R.M. Marcé, P.A.G. Cormack, D.C. Sherrington, F. Borrull, Direct determination of ciprofloxacin by mass spectrometry after a two-step solid-phase extraction using a molecularly imprinted polymer, J. Sep. Sci., 29 (2006) 1230-1236.

[18] K. Balamurugan, K. Gokulakrishnan, T. Prakasam, Preparation and evaluation of molecularly imprinted polymer liquid chromatography column for the separation of ephedrine enantiomers, Arabian J. Chem., DOI http://dx.doi.org/10.1016/j.arabjc.2011.06.024(2011).

[19] K. Tang, X. Gu, Q. Luo, S. Chen, L. Wu, J. Xiong, Preparation of molecularly imprinted polymer for use as SPE adsorbent for the simultaneous determination of five sulphonylurea herbicides by HPLC, Food Chem., 150 (2014) 106-112. 
[20] M. Ramamoorthy, M. Ulbricht, Molecular imprinting of cellulose acetate-sulfonated polysulfone blend membranes for Rhodamine B by phase inversion technique, J. Membr. Sci., 217 (2003) 207-214.

[21] K. Reimhult, K. Yoshimatsu, K. Risveden, S. Chen, L. Ye, A. Krozer, Characterization of QCM sensor surfaces coated with molecularly imprinted nanoparticles, Biosens. Bioelectron., 23 (2008) 1908-1914.

[22] L. Chen, S. Xu, J. Li, Recent advances in molecular imprinting technology: current status, challenges and highlighted applications, Chemical Society reviews, 40 (2011) 2922-2942.

[23] X. Ding, P.A. Heiden, Recent Developments in Molecularly Imprinted Nanoparticles by Surface Imprinting Techniques, Macromol. Mater. Eng., 299 (2014) 268-282.

[24] C. Folch-Cano, M. Yazdani-Pedram, C. Olea-Azar, Inclusion and Functionalization of Polymers with Cyclodextrins: Current Applications and Future Prospects, Molecules, 19 (2014) 14066-14079.

[25] M.C. Pietrogrande, G. Basaglia, GC-MS analytical methods for the determination of personal-care products in water matrices, TrAC, Trends Anal. Chem., 26 (2007) 1086-1094.

[26] J.L. Tadeo, C. Sánchez-Brunete, B. Albero, A.I. García-Valcárcel, R.A. Pérez, Analysis of emerging organic contaminants in environmental solid samples, Cent. Eur. J. Chem., 10 (2012) 480-520.

[27] C. Haman, X. Dauchy, C. Rosin, J.-F. Munoz, Occurrence, fate and behavior of parabens in aquatic environments: A review, Water Research, 68 (2015) 1-11.

[28] Y. Luo, W. Guo, H.H. Ngo, L.D. Nghiem, F.I. Hai, J. Zhang, S. Liang, X.C. Wang, A review on the occurrence of micropollutants in the aquatic environment and their fate and removal during wastewater treatment, Sci. Total Environ., 473-474 (2014) 619-641.

[29] L. Chen, S. Xu, J. Li, Recent advances in molecular imprinting technology: current status, challenges and highlighted applications, Chemical Society Reviews, 40 (2011) 2922-2942.

[30] P.A.G. Cormack, A.Z. Elorza, Molecularly imprinted polymers: synthesis and characterisation, J. Chromatogr. B, 804 (2004) 173-182.

[31] D.A. Spivak, Optimization, evaluation, and characterization of molecularly imprinted polymers, Adv. Drug Delivery Rev., 57 (2005) 1779-1794.

[32] A. Ellwanger, C. Berggren, S. Bayoudh, C. Crecenzi, L. Karlsson, P.K. Owens, K. Ensing, P. Cormack, D. Sherrington, B. Sellergren, Evaluation of methods aimed at complete removal of template from molecularly imprinted polymers, Analyst, 126 (2001) 784-792.

[33] R.A. Lorenzo, A.M. Carro, C. Alvarez-Lorenzo, A. Concheiro, To remove or not to remove? The challenge of extracting the template to make the cavities available in Molecularly Imprinted Polymers (MIPs), Int J Mol Sci, 12 (2011) 4327-4347.

[34] R. Liang, L. Kou, Z. Chen, W. Qin, Molecularly imprinted nanoparticles based potentiometric sensor with a nanomolar detection limit, Sensors and Actuators B: Chemical, 188 (2013) 972-977.

[35] Z. Zhongbo, J. Hu, Selective removal of estrogenic compounds by molecular imprinted polymer (MIP), Water Research, 42 (2008) 4101-4108.

[36] M. Lopez-Nogueroles, S. Lordel-Madeleine, A. Chisvert, A. Salvador, V. Pichon, Development of a selective solid phase extraction method for nitro musk compounds in environmental waters using a molecularly imprinted sorbent, Talanta, 110 (2013) 128-134.

[37] V. Pichon, K. Haupt, Affinity Separations on Molecularly Imprinted Polymers with Special Emphasis on Solid-Phase Extraction, J. Liq. Chromatogr. Related Technol., 29 (2006) 989-1023.

[38] G. Vasapollo, R.D. Sole, L. Mergola, M.R. Lazzoi, A. Scardino, S. Scorrano, G. Mele, Molecularly imprinted polymers: present and future prospective, Int J Mol Sci, 12 (2011) 59085945.

[39] V. Kandimalla, H. Ju, Molecular imprinting: a dynamic technique for diverse applications in analytical chemistry, Anal. Bioanal.Chem., 380 (2004) 587-605.

[40] J. He, S. Chen, Y. Jiang, Y. Shen, J. Zhu, H. Wei, H. Zhang, K. Lu, Preparation and selective recognition of a novel solid-phase microextraction fiber combined with molecularly imprinted polymers for the extraction of parabens in soy sample, J. Sep. Sci., 35 (2012) 308-314. 
[41] S. Shi, J. Guo, Q. You, X. Chen, Y. Zhang, Selective and simultaneous extraction and determination of hydroxybenzoic acids in aqueous solution by magnetic molecularly imprinted polymers, Chem. Eng. J., 243 (2014) 485-493.

[42] G. Karasová, J. Lehotay, J. Sádecká, I. Skačáni, M. Lachová, Selective extraction of derivates of p-hydroxy-benzoic acid from plant material by using a molecularly imprinted polymer, J. Sep. Sci., 28 (2005) 2468-2476.

[43] L. Núñez, E. Turiel, A. Martin-Esteban, J.L. Tadeo, Molecularly imprinted polymer for the extraction of parabens from environmental solid samples prior to their determination by high performance liquid chromatography-ultraviolet detection, Talanta, 80 (2010) 1782-1788.

[44] R. Gao, X. Kong, F. Su, X. He, L. Chen, Y. Zhang, Synthesis and evaluation of molecularly imprinted core-shell carbon nanotubes for the determination of triclosan in environmental water samples, J. Chromatogr. A, 1217 (2010) 8095-8102.

[45] A.G. Mayes, K. Mosbach, Molecularly Imprinted Polymer Beads: Suspension Polymerization Using a Liquid Perfluorocarbon as the Dispersing Phase, Analytical Chemistry, 68 (1996) 3769-3774.

[46] W.J. Cheong, S.H. Yang, F. Ali, Molecular imprinted polymers for separation science: A review of reviews, Journal of Separation Science, 36 (2013) 609-628.

[47] J.E. Lofgreen, G.A. Ozin, Controlling morphology and porosity to improve performance of molecularly imprinted sol-gel silica, Chemical Society Reviews, 43 (2014) 911-933.

[48] N. Pérez-Moral, A.G. Mayes, Comparative study of imprinted polymer particles prepared by different polymerisation methods, Analytica Chimica Acta, 504 (2004) 15-21.

[49] K.S. Verma, K. Xia, Analysis of triclosan and triclocarban in soil and biosolids using molecularly imprinted solid phase extraction coupled with HPLC-UV, Journal Of AOAC International, 93 (2010) 1313-1321.

[50] H. Li, H. Guan, H. Dai, Y. Tong, X. Zhao, W. Qi, S. Majeed, G. Xu, An amperometric sensor for the determination of benzophenone in food packaging materials based on the electropolymerized molecularly imprinted poly-o-phenylenediamine film, Talanta, 99 (2012) 811-815.

[51] T. Osawa, K. Shirasaka, T. Matsui, S. Yoshihara, T. Akiyama, T. Hishiya, H. Asanuma, M. Komiyama, Importance of the Position of Vinyl Group on $\beta$-Cyclodextrin for the Effective Imprinting of Amino Acid Derivatives and Oligopeptides in Water, Macromolecules, 39 (2006) 2460-2466.

[52] S.-h. Song, K. Shirasaka, M. Katayama, S. Nagaoka, S. Yoshihara, T. Osawa, J. Sumaoka, H. Asanuma, M. Komiyama, Recognition of Solution Structures of Peptides by Molecularly Imprinted Cyclodextrin Polymers, Macromolecules, 40 (2007) 3530-3532.

[53] X.-A. Ton, V. Acha, K. Haupt, B. Tse Sum Bui, Direct fluorimetric sensing of UV-excited analytes in biological and environmental samples using molecularly imprinted polymer nanoparticles and fluorescence polarization, Biosens. Bioelectron., 36 (2012) 22-28.

[54] F. Horemans, A. Weustenraed, D. Spivak, T.J. Cleij, Towards water compatible MIPs for sensing in aqueous media, Journal of Molecular Recognition, 25 (2012) 344-351.

[55] M.Á. Lorenzo, A. Sánchez Arribas, M. Moreno, E. Bermejo, M. Chicharro, A. Zapardiel, Determination of butylparaben by adsorptive stripping voltammetry at glassy carbon electrodes modified with multi-wall carbon nanotubes, Microchemical Journal, 110 (2013) 510-516.

[56] J. He, Y. Shen, S. Chen, H. Wei, J. Zhu, L. You, K. Lu, Preparation and evaluation of molecularly imprinted microspheres for solid-phase extraction of 1,4-hydroxybenzoic acid esters in soy, J. Sep. Sci., 34 (2011) 2739-2744.

[57] A. Beltran, R.M. Marcé, P.A.G. Cormack, F. Borrull, Synthetic approaches to parabens molecularly imprinted polymers and their applications to the solid-phase extraction of river water samples, Analytica Chimica Acta, 677 (2010) 72-78.

[58] B. Kasprzyk-Hordern, R.M. Dinsdale, A.J. Guwy, The occurrence of pharmaceuticals, personal care products, endocrine disruptors and illicit drugs in surface water in South Wales, UK, Water Res., 42 (2008) 3498-3518.

[59] J. Regueiro, E. Becerril, C. Garcia-Jares, M. Llompart, Trace analysis of parabens, triclosan and related chlorophenols in water by headspace solid-phase microextraction with in situ 
derivatization and gas chromatography-tandem mass spectrometry, J. Chromatogr. A, 1216 (2009) 4693-4702.

[60] N. Lozano, C.P. Rice, M. Ramirez, A. Torrents, Fate of triclosan in agricultural soils after biosolid applications, Chemosphere, 78 (2010) 760-766.

[61] G.-G. Ying, R.S. Kookana, Triclosan in wastewaters and biosolids from Australian wastewater treatment plants, Environ. Int., 33 (2007) 199-205.

[62] V. Suryanarayanan, C.-T. Wu, K.-C. Ho, Molecularly Imprinted Electrochemical Sensors, Electroanalysis, 22 (2010) 1795-1811.

[63] A. Hulanicki, S. Glab, F. Ingman, Chemical sensors definitions and classification, Pure Appl. Chem., 63 (1991) 1247-1250.

[64] W.J. Cheong, F. Ali, J.H. Choi, J.O. Lee, K. Yune Sung, Recent applications of molecular imprinted polymers for enantio-selective recognition, Talanta, 106 (2013) 45-59.

[65] Y. Liu, Q.-J. Song, L. Wang, Development and characterization of an amperometric sensor for triclosan detection based on electropolymerized molecularly imprinted polymer, Microchem. J., 91 (2009) 222-226.

[66] Y. Wang, Y. Cao, C. Fang, Q. Gong, Electrochemical sensor for parabens based on molecular imprinting polymers with dual-templates, Analytica Chimica Acta, 673 (2010) 145-150.

[67] M.B. Gholivand, M. Shamsipur, S. Dehdashtian, H.R. Rajabi, Development of a selective and sensitive voltammetric sensor for propylparaben based on a nanosized molecularly imprinted polymer-carbon paste electrode, Materials Science and Engineering: C, 36 (2014) 102-107.

[68] J.A. García-Calzón, M.E. Díaz-García, Characterization of binding sites in molecularly imprinted polymers, Sens. Actuators, B, 123 (2007) 1180-1194.

[69] L.Y. Hu, R.A. Orwoll, Molecular Imprinting by 4-Hydroxybenzoic Acid: A Two-Site Model, Sep. Sci. Technol., 45 (2010) 2337-2344.

[70] B.W. Sun, Y.Z. Li, W.B. Chang, Molecularly imprinted polymer using-p-hydroxybenzoic acid, p-hydroxyphenylacetic acid and p-hydroxyphenylpropionic acid as templates, J. Mol. Recognit., 14 (2001) 388-392.

[71] M. Meng, Z. Wang, L. Ma, M. Zhang, J. Wang, X. Dai, Y. Yan, Selective Adsorption of Methylparaben by Submicrosized Molecularly Imprinted Polymer: Batch and Dynamic Flow Mode Studies, Ind. Eng. Chem. Res., 51 (2012) 14915-14924.

[72] S.F. Abdul Raof, S. Mohamad, M.R. Abas, Synthesis and Evaluation of Molecularly Imprinted Silica Gel for 2-Hydroxybenzoic Acid in Aqueous Solution, Int J Mol Sci, 14 (2013) 5952-5965.

[73] C. Radovan, D. Cinghită, F. Manea, M. Mincea, C. Cofan, V. Ostafe, Electrochemical Sensing and Assessment of Parabens in Hydro- Alcoholic Solutions and Water Using a BoronDoped Diamond Electrode, Sensors, 8 (2008) 4330-4349.

[74] W. Wang, Y. Wang, J. Zhang, Q. Chu, J. Ye, Simultaneous determination of electroactive and non-electroactive food preservatives by novel capillary electrophoresis with amperometric detection, Anal. Chim. Acta, 678 (2010) 39-43.

[75] J. Yang, P. Wang, X. Zhang, K. Wu, Electrochemical Sensor for Rapid Detection of Triclosan Using a Multiwall Carbon Nanotube Film, J. Agric. Food. Chem., 57 (2009) 9403-9407.

[76] H. Dai, G. Xu, L. Gong, C. Yang, Y. Lin, Y. Tong, J. Chen, G. Chen, Electrochemical detection of triclosan at a glassy carbon electrode modifies with carbon nanodots and chitosan, Electrochim. Acta, 80 (2012) 362-367.

[77] M.T. Laranjeira, F. de Lima, S.C. Oliveira, V.S. Ferreira, R.T.S. Oliveira, Analytical Determination of Benzophenone-3 in Sunscreen Preparations Using Boron-Doped Diamond Electrodes, Am. J. Anal. Chem., 2 (2011) 383-391. 


\section{Graphical Abstract}
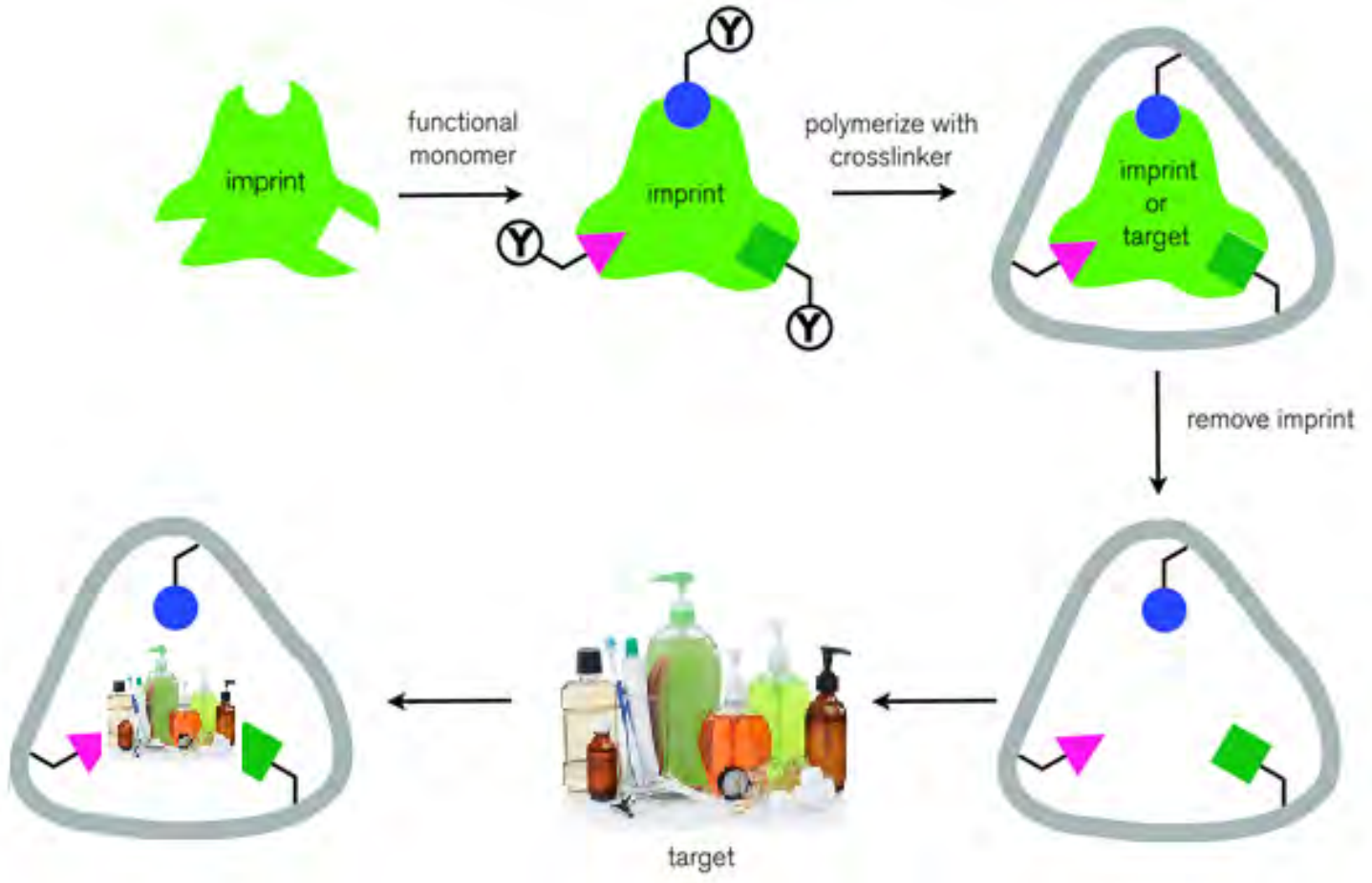
Figure 1

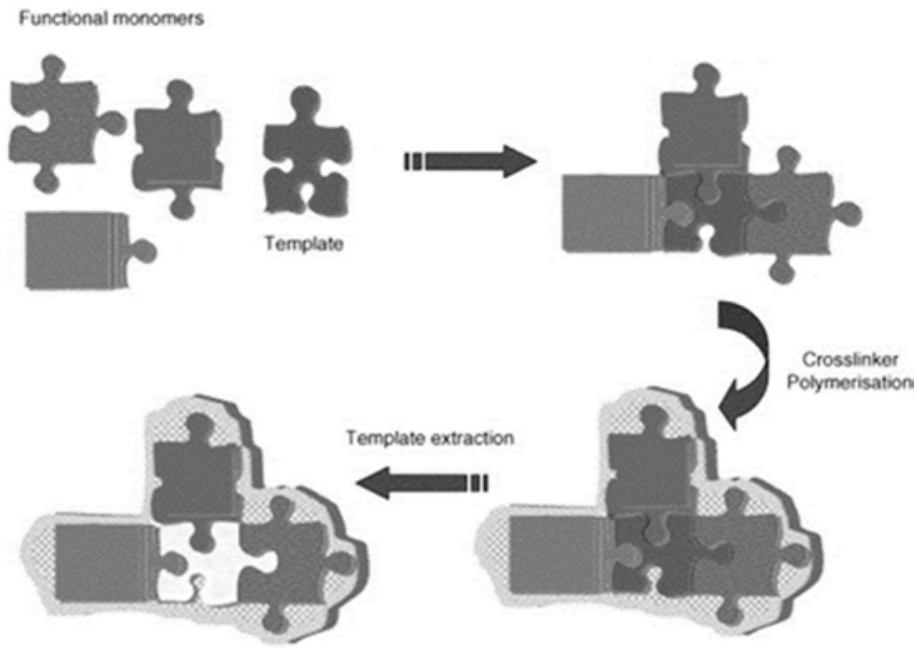


Figure 2

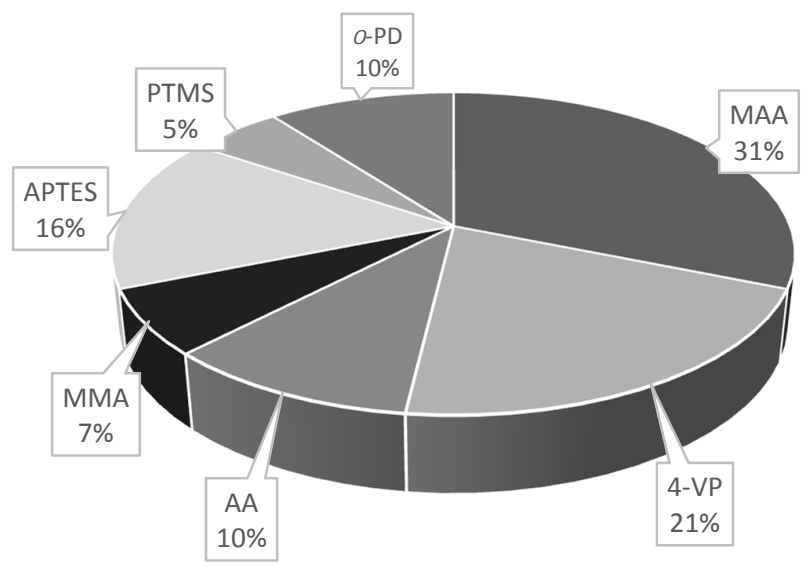


Figure 3

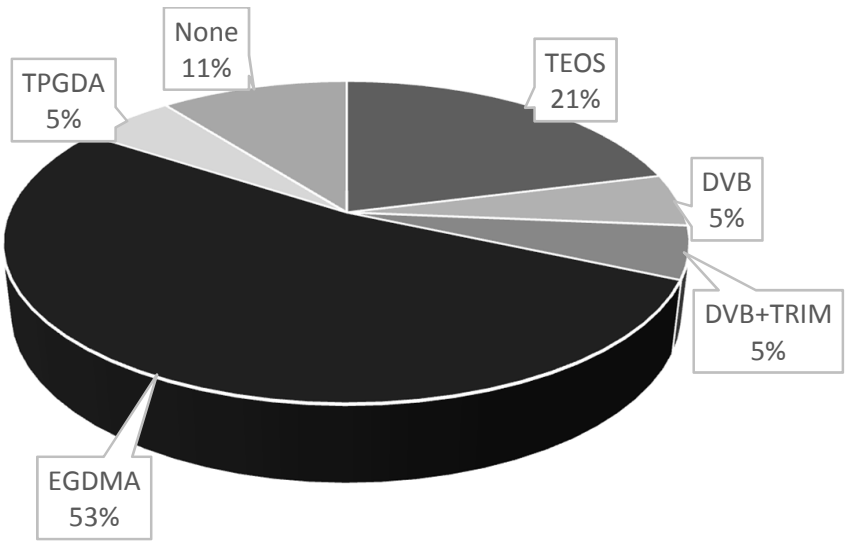


Figure 4

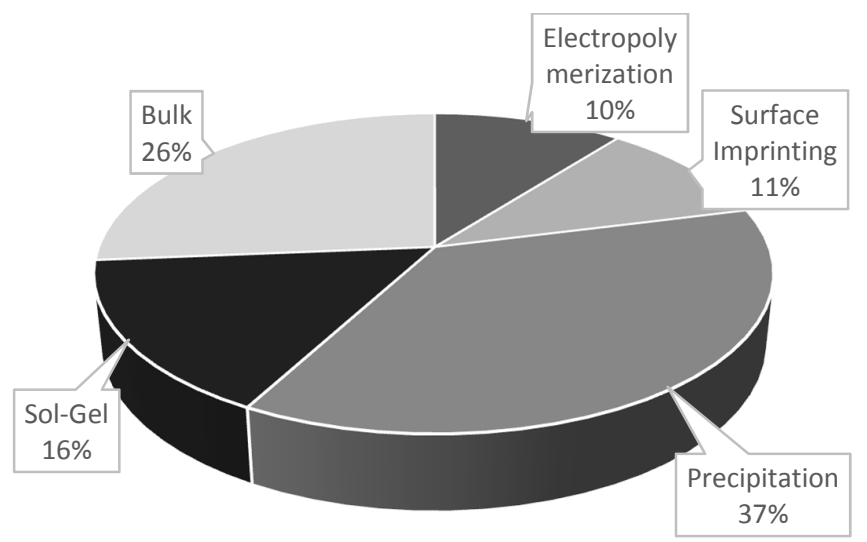


Figure 5

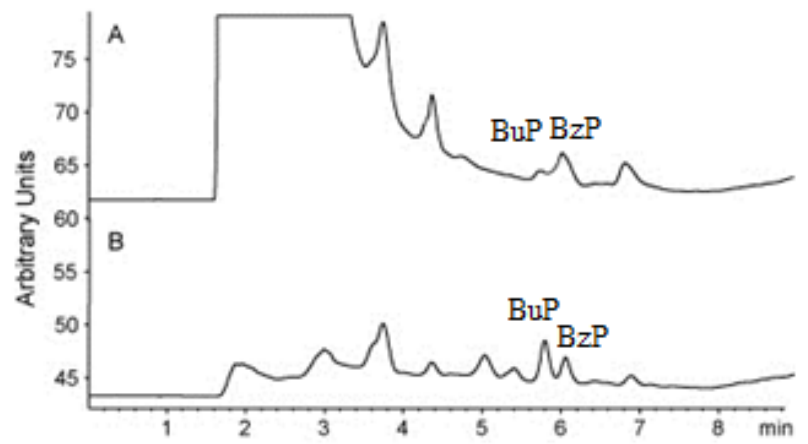

